\title{
Du subir à l'agir. Au fil des intelligences citoyennes
}

\section{Majo Hansotte}

\section{(2) OpenEdition}

Journals

Édition électronique

URL : https://journals.openedition.org/ere/6393

DOI : $10.4000 /$ ere.6393

ISSN : 2561-2271

Éditeur

Centr'ERE

Référence électronique

Majo Hansotte, «Du subir à l'agir. Au fil des intelligences citoyennes », Éducation relative à l'environnement [En ligne], Volume 16 - 1 | 2021, mis en ligne le 10 mars 2021, consulté le 17 septembre 2021. URL : http://journals.openedition.org/ere/6393 ; DOI : https://doi.org/10.4000/ere 6393

Ce document a été généré automatiquement le 17 septembre 2021.

\section{(ब) (1) \&}

La revue Éducation relative à l'environnement est mise à disposition selon les termes de la Licence Creative Commons Attribution - Pas d'Utilisation Commerciale 4.0 International. 


\title{
Du subir à l'agir. Au fil des intelligences citoyennes
}

\author{
Majo Hansotte
}

1 Que nous est-il arrivé? Désastres écologiques, pandémies, migrations, violences, inégalités. Nous sommes confrontés à une bifurcation historique: s'annonce une aventure indéterminée où l'ultime responsabilité citoyenne sera celle répondre à l'urgence de préserver le monde vivant.

2 Or déni ou démoralisation se manifestent actuellement de manière dominante, malgré l'émergence de diverses formes de résistance et de réaction, comme si nous, les humains, potentiellement dotés d'un pouvoir d'agir, étions dans l'incapacité de départager, d'arbitrer, de réguler pour changer le cours des choses.

Cette impuissance à dire, à réguler, est-ce la fin des conquêtes propres aux révolutions démocratiques du $18^{\mathrm{e}}$ siècle et aux mouvements sociaux des $19^{\mathrm{e}}$ et $20^{\mathrm{e}}$ siècles?

4 Partir de ce diagnostic est indispensable pour aborder les exigences méthodologiques d'une intelligence collective porteuse d'un devenir, laquelle intelligence devrait s'appréhender comme une nécessaire épreuve commune.

Dans un ouvrage antérieur (Hansotte, 2008), j'ai fait apparaître les modalités d'émergence de cette conquête de la parole humaine qu'est la citoyenneté, entendue comme puissance de transformation des conditions d'existence. Et cette conquête a une prétention universelle dans la mesure où tous les humains sont doués de parole et peuvent être amenés à faire l'expérience de l'Injuste, impliquant une espérance de plus de Justice.

Dire le Juste et l'Injuste, à travers le déploiement des personnes grammaticales Je, Tu, Nous, Nous Tous et Toutes, présuppose de ne pas attendre qu'une autorité surplombante (Dieu, Roi, Finance, Marché) l'énonce et le détermine. Comme nous le verrons, il est question ici d'activer des Intelligences citoyennes que j'ai dégagées et formalisées. 


\section{La citoyenneté, un acte de parole}

7 La conquête de la citoyenneté s'appuie sur une prise de parole en tant qu'acte, laquelle dépasse la vie personnelle ou laborieuse pour investir l'espace public. Ce terme est une métaphore à ne pas entendre comme lieu public, mais comme une dimension de l'expérience humaine, motrice de la démocratie, source d'actions collectives politiques (au sens de la Polis, la cité commune des humains). Le contexte indispensable au déploiement de cet espace public est une vie citoyenne libre et autonome, fondée sur l'engagement volontaire.

8 Une telle dynamique configure la démocratie comme un régime en trois niveaux, lequel ne met pas face à face, comme dans l'Ancien Régime, des gouvernants et des gouvernés, mais inscrit, au cœur de son fonctionnement, le déploiement d'un espace public, aujourd'hui local et mondial. Le pouvoir n'appartient à personne, il est une fonction provisoire que nous prêtons à des mandataires et dont nous, les citoyens et citoyennes, sommes la source.

9 Notons qu'aujourd'hui, si l'espace public citoyen est à l'œuvre dans les régimes démocratiques, où il est en principe respecté comme un droit inaliénable, il est aussi à l'œuvre dans de nombreux lieux et au sein de nombreux régimes où il est persécuté et où s'y engager fait encourir des répressions violentes.

10 Les mouvements sociaux des $19^{\mathrm{e}}$ et $20^{\mathrm{e}}$ siècles (mouvements ouvriers, mouvements pour les droits civiques, luttes pour la décolonisation, mouvements de femmes, mouvements paysans, mouvements écologiques, mouvements étudiants, mouvements pacifistes, anti-impérialistes, etc.) ont enrichi la conception de l'espace public citoyen, en le pratiquant comme une scénographie conflictuelle, mettant en scène des affrontements pour les rendre agissants et sensibles, c'est-à-dire pouvant faire leur chemin dans le cœur et la tête d'autres humains.

11 La protestation passe du verbe au corps, entendu comme parole en acte. Avec le défi périlleux et permanent pour les citoyens et citoyennes de mener une offensive forte tout en évitant la violence haineuse (Hansotte, 2013, 2019).

12 Cette mutation qui associe verbe et corps confère une double perspective à l'espace public: à la fois processus horizontal, culturel et symbolique, de transformation réciproque des mentalités et dynamique verticale d'affrontement des pouvoirs quels qu'ils soient.

$13 \mathrm{Au}$ sein des régimes de tridimensionnalité démocratique ont été obtenus, en particulier dans les démocraties sociales avancées, des droits précieux, à travers des interactions constructives entre États de Droit et espace public citoyen (Tableau 1).

14 Ont donc été inventées, au cours des $19^{\mathrm{e}}$ et $20^{\mathrm{e}}$ siècles, des modalités de régulation des violences sociales et humaines, via des institutions, des lois et grâce à des collectifs reconnus, dont les syndicats notamment. Bien sûr, les évolutions technologiques ont régulièrement devancé la régulation sociale, mais celle-ci trouvait le temps et les ressources pour se construire. 
Tableau 1 : Les démocraties avancées se sont déployées en 3 sphères interactives

\section{LES ÉTATS DE DROIT}

Les mandataires en charge de la gestion et de I'arbitrage politiques

\begin{tabular}{|c|c|c|}
\hline \multicolumn{3}{|c|}{$\Delta$} \\
\hline \multicolumn{3}{|c|}{$\begin{array}{l}\text { UN ESPACE PUBLIC LOCAL ET MONDIAL AU COEUR DES DÉMOCRATIES } \\
\text { Avec la parole, le corps et I'action, Je, Tu, Nous } \\
\text { proposons, revendiquons, imaginons, refusons, inventons, analysons, } \\
\text { débattons, projetons, anticipons... pour nous TOUS et TOUTES }\end{array}$} \\
\hline \multicolumn{3}{|c|}{$\nabla \quad \Delta$} \\
\hline $\begin{array}{l}\text { LA VIE PERSONNELLE } \\
\text { Relations affectives, } \\
\text { famille, loisirs... } \\
\text { croyances, } \\
\text { pratiques culturelles } \\
\text { et sociales particulières }\end{array}$ & $\begin{array}{c}\text { LA VIE CITOYENNE } \\
\text { Libres associations } \\
\text { Libres information } \\
\text { et parole } \\
\text { Libres circulation des idées, } \\
\text { expression, création... } \\
\text { Contexte } \\
\text { de l'espace public }\end{array}$ & $\begin{array}{c}\text { LA VIE LABORIEUSE } \\
\text { Contrats et entreprenariat } \\
\text { Marché, services, } \\
\text { production ... } \\
\text {................. } \\
\text { Son envers : Le Non-emploi } \\
\end{array}$ \\
\hline
\end{tabular}

\section{Où en sommes-nous aujourd'hui ?}

15 Un sentiment d'impuissance domine, donnant l'impression que l'on ne peut plus avoir prise, ni sur nous-mêmes en tant que société ni sur le cours des choses, qu'il s'agisse de la protection de la vie privée, des droits sociaux, de la santé, de l'avenir de l'humain, de la robotisation, du nucléaire, de la pollution des terres et des mers, de la contamination alimentaire, de l'accueil des migrants, des sources énergétiques, des politiques climatiques, etc.

Tout se passe comme si les acteurs et actrices démocratiques, mandataires et citoyens/ citoyennes, n'arrivaient plus à réguler les évolutions. Parmi d'autres, Stiegler craint, si nous ne réagissons pas, que soit impossible la régulation démocratique de notre devenir commun. Cette impossibilité est liée en grande partie à ce qu'il appelle la «gouvernementalité algorithmique » reposant sur une instrumentalisation et selon ses mots une "réticulation" (une mise en réseaux) systématique des relations interindividuelles et transindividuelles, au service de la data economy, basée sur le calcul intensif de données massives ou big data (Stiegler, 2016, p. 15 à 25).

17 Selon lui, la réticulation numérique, à une vitesse foudroyante, pénètre, envahit, parasite les relations sociales et les neutralise ou les annihile de l'intérieur, les prenant de vitesse et les phagocytant, stérilisant et détruisant tout ce qui procédait de la culture et de la vie sociale. Ce phénomène, Stiegler le nomme « disruption ».

18 La disruption ne se réduit pas à la vitesse de la pénétration numérique ; elle désigne plus largement toutes les formes d'entreprises que nous n'arrivons plus à réguler. Elle est ce qui va plus vite que toute volonté, individuelle aussi bien que collective. 
19 L'on songe à l'ubérisation des emplois, faisant voler en éclats les droits sociaux et mettant hors-jeu les concertations syndicales. De même, la pandémie Covid 19 a renforcé la norme de plus en plus dominante du télétravail, dont les potentialités sont louées mais sans que nous ayons pu en déterminer les pièges et les aliénations cachées. Plus largement, cette pandémie a accéléré la dématérialisation et la mise en plateformes numériques des services publics, de l'enseignement et des soins de santé, faisant l'économie très rentable des lieux concrets de vie et d'échanges. Quel en sera le prix humain?

20 Pourrons-nous arbitrer ces évolutions, empêcher leur inéluctabilité, tenter de les rendre plus justes et humaines? La disruption prend de vitesse les organisations sociales, qui ne parviennent à appréhender une mutation que lorsqu'elle est déjà devenue du passé. La volonté collective, d'où qu'elle vienne, est par avance obsolète, elle arrive toujours trop tard. Comment sortir de cette impasse?

21 Stiegler évoque, en lien avec cette perte de pouvoir régulateur, une misère symbolique liée à une déterritorialisation radicale de l'être humain qui, dans l'impossibilité de mettre en suspens les flux brutaux de l'existence, peut être poussé à la démoralisation, voire au suicide (Stiegler, 2016, p. 15 à 57 et 319 à 321).

22 Si la plupart des sociétés fondées sur le capitalisme ont été capables de domestiquer ce système durant la période des 30 glorieuses, inventant un modèle économique reposant sur un compromis social, rester dans la logique d'aménagement du capitalisme n'est plus pertinent à long terme aujourd'hui, notamment du strict point de vue écologique, parce qu'un tel aménagement du capitalisme supposait une croissance forte et continue.

Or les flux brutaux de la data economy ne vont pas vers la sortie du capitalisme, bien au contraire. Symptomatique de cette évolution, l'aggravation permanente de la crise écologique résulte d'une sur-appropriation, le degré d'appropriation des ressources naturelles outrepassant largement leur rythme de renouvellement.

24 Cette crise écologique s'accompagne d'une crise politique, qui mine les conditions mêmes de la démocratie, notamment en réduisant constamment les ressources temporelles disponibles pour construire des décisions politiques justes. Le phénomène d'accélération sociale favorise donc le transfert de la décision politique vers des sphères plus rapides : privatisation, dérégulation, judiciarisation, etc.

Et plus largement, depuis les années 1980, on a assisté à la déstabilisation des interactions entre États de Droit et mouvements sociaux, autrement dit, entre États de Droit et espace public. L'Europe de l'Ouest en particulier vit une lente et irrésistible érosion des conquêtes sociales et des interactions productives entre États et mouvements sociaux, à l'œuvre dans l'après-guerre. Et cette érosion n'est pas propre à l'Europe. En résultent un peu partout dans les démocraties un désenchantement démocratique et une méfiance à l'égard des mandataires politiques comme à l'égard des modalités de leurs élections, un divorce dont le Tableau 2 rend compte. 


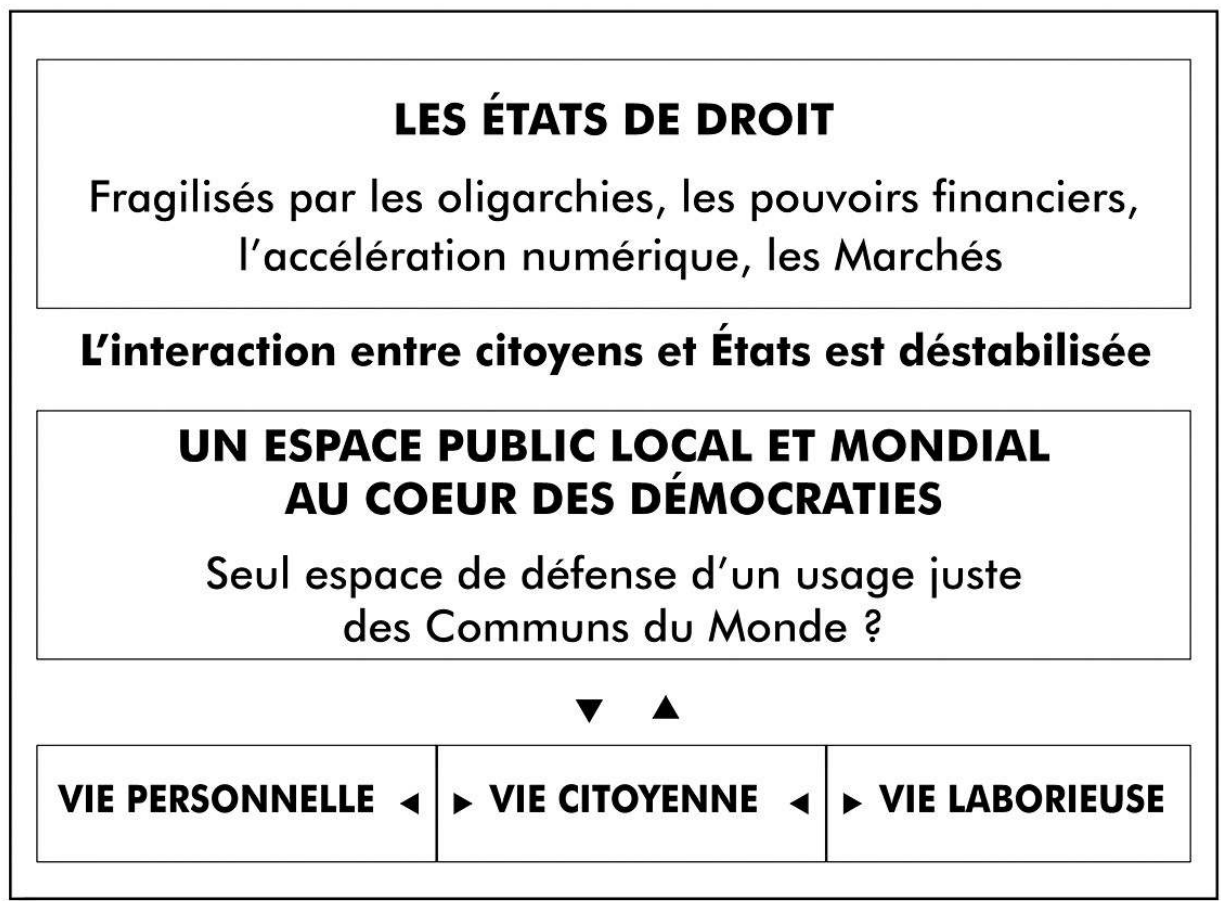

Le tragique affaiblissement des États de Droit est gros de menaces : montée de la haine, du racisme, multiplication d'autocrates dits populistes, extrémismes, affirmation absolue de l'individu contre les processus solidaires. Il devient essentiel que les engagements citoyens relaient avec force les questions touchant au Juste et à l'Injuste.

Comment réinventer une nouvelle forme de puissance publique? Le Tableau 2 fait apparaitre que nous sommes dans une bifurcation. S'annonce en effet une aventure indéterminée où l'espace public local et mondial deviendrait la principale instance garante de la question du Juste et de l'Injuste (Hansotte, 2013, 2019). Cette bifurcation engage plus que jamais notre responsabilité citoyenne, avec l'impérieuse nécessité de disposer de méthodes et de processus pour assumer ce défi.

\section{Des visées communes pour construire une volonté collective}

Comment voir émerger un jour une instance citoyenne, une instance productrice d'une intelligence collective, reconnue comme valide et légitime, qui disposerait d'un réel pouvoir d'intervention dans la sphère politique? Plusieurs attitudes de base sont à mettre en œuvre.

\section{Refuser le piège du hors temps et du hors sol}

Comment retrouver les ressources temporelles disponibles et déployer les méthodes collectives nécessaires pour coconstruire des décisions politiques justes? Tel est l'enjeu de la démarche des intelligences citoyennes. 
30 Dans la mesure où la vitesse a partie liée avec le capitalisme, toute accélération se fait toujours au détriment des plus faibles et menace notre devenir commun. Il est donc primordial de prendre le temps de la pensée et de l'action. Nous pouvons refuser le hors temps comme le hors sol, sortir de l'aplatissement total de l'espace et du temps.

31 Il faut inventer un nouvel âge de l'intelligence collective, en s'appuyant bien sûr aussi sur le numérique comme organe de publication, mais surtout en activant, par différents registres, les potentialités d'un espace public local et mondial.

32 Voir la citoyenneté comme une communication/conversation permanente est désastreux, car une telle vision nous entretient dans un relativisme, où tous les propos se valent, sans aucun arbitrage rigoureux. Un tel relativisme fait le lit des extrémismes en tout genre et il encourage une passivité consommatoire : nous dormons pendant que l'arbitraire s'installe.

33 Le propre de la démarche des intelligences citoyennes est de privilégier des espaces de parole où les personnes refont corps et ne sont pas réduites à des messages virtuels. Retrouver le temps et le pouvoir personnel de dire, de refuser ou de faire, soit «le pouvoir de»; mais aussi retrouver l'intelligence collective du "pouvoir avec», le pouvoir collectif d'entreprendre un combat, une action. Permettre à des collectifs de construire des rapports offensifs, d'agir à travers des intelligences citoyennes.

La visée poursuivie dans les intelligences citoyennes est celle de la transformation sociale qui se distingue de la réparation sociale. Ainsi notamment, l'exigence de transformation sociale ne se confond pas avec la judiciarisation des litiges ni avec les règlements de compte interpersonnels. Il ne s'agit pas, pour un collectif soucieux de déployer les intelligences citoyennes, de dénoncer une personne physique ou morale particulière en vue d'une vengeance médiatique par exemple. Bien sûr, à condition de ne pas confondre les visées, un collectif, outre l'agir citoyen, peut soutenir à titre fraternel une personne en quête de réparation individuelle.

35 Toutefois, l'enjeu central est de chercher à énoncer un devenir juste pour tous et toutes, de formuler une énonciation porteuse d'actions et d'un devenir collectif. Il ne s'agit pas de provoquer le Buzz. Certaines campagnes médiatiques peuvent servir de contexte favorable, d'adjuvants ou d'outils puissants aux mouvements citoyens de fond, à condition de ne pas s'enfermer dans le battage médiatique ne portant vers aucun devenir.

36 Il ne faut pas accepter d'être les otages captifs et impuissants de Facebook et de ses actionnaires, par exemple, mais rester acteurs et actrices responsables sur le terrain d'un travail concret de transformation des pratiques. La sphère médiatique et la sphère de l'espace public citoyen entretiennent des interactions fortes, dans certains cas très constructives et mobilisatrices, mais ne se confondent pas.

37 Il importe donc de faire la distinction entre l'espace public citoyen dont la finalité est d'imposer les questions touchant au Juste et à l'Injuste et les espaces médiatiques, en particulier numériques, dont la finalité est d'être diffusés, consommés et qui en conséquence peuvent servir mais aussi menacer l'espace public (Tableaux 3 et 4). Car la perspective citoyenne est d'abord politique et non pas d'abord médiatique; elle fait vivre tous les processus mettant en mouvement des gens, autour d'une exigence ou d'un problème à traiter, processus au cours desquels les relations sociales se trouvent modifiées. De même se modifie la représentation que chacun a de soi et des autres (Hansotte, 2013,2019). 
Tableau 3 : Les fonctions de l'espace public au service du Juste et de l'Injuste

\begin{tabular}{|l|l|}
\hline \multicolumn{1}{|c|}{$\begin{array}{c}\text { ESPACE PUBLIC } \\
\text { Fonction verticale } \\
\text { de contre-pouvoir }\end{array}$} & $\begin{array}{c}\text { ESPACE PUBLIC } \\
\text { Fonction horizontale de } \\
\text { transformations réciproques }\end{array}$ \\
$\begin{array}{l}\text { 1. Vigilance critique à l'égard des } \\
\text { mandataires d'ici et d'ailleurs }\end{array}$ & $\begin{array}{l}\text { 1. Partage de paroles, de récits, de } \\
\text { savoirs et méthodologies de l'action }\end{array}$ \\
$\begin{array}{l}\text { 2. Actions de résistances face aux } \\
\text { pouvoirs politiques, économiques, } \\
\text { financiers, religieux, médiatiques... }\end{array}$ & $\begin{array}{l}\text { 2. Déconstruction des codes dominants } \\
\text { et des manipulations }\end{array}$ \\
$\begin{array}{l}\text { 3. Interpellations publiques des } \\
\text { pouvoirs arbitraires }\end{array}$ & $\begin{array}{l}\text { 3. Transmission des luttes sociales, } \\
\text { des conquêtes historiques et des } \\
\text { référents démocratiques }\end{array}$ \\
$\begin{array}{l}\text { 4. Mobilisations conflictuelles autour } \\
\text { du Juste et de l'Injuste }\end{array}$ & $\begin{array}{l}\text { 4. Elargissement des mentalités et } \\
\text { brassage des langages ou symboles }\end{array}$ \\
$\begin{array}{l}\text { 5. Scénographies de l'affrontement, } \\
\text { expression des conflits... }\end{array}$ & $\begin{array}{l}\text { 5. Invention d'utopies et construction } \\
\text { collective d'interventions créatives }\end{array}$ \\
\hline
\end{tabular}

Tableau 4 : Les espaces médiatiques servent et desservent l'espace public citoyen

\section{Ils servent l'espace public}

1. Réseaux sociaux et mobilisations citoyennes

2. Circulation des témoignages et des vécus

3. Mentalités élargies

4. Informations, diffusion des faits, investigations, dévoilements...

5. Questionnements critiques

6. Diffusion d'alternatives sociales, économiques, écologiques...

7. Partage des cultures, des esthétiques et des savoirs

\section{Ils nuisent à l'espace public}

1. Imposture de l'opinion publique

2. Manipulations rhétoriques et communications sociales déformées

3. Réseaux sociaux et bulles cognitives : post-vérité

4. Régime de la rumeur, politiquepeople, faux événements, buzz

5. Big data et algorithmes : manipulation permanente

6. Consommation plutôt qu'action

7. Surveillance panoptique, menace sur la vie privée 


\section{Articuler les Communs}

Dans le contexte de crises brutales et multiples que nous vivons, il est essentiel de reconstruire un imaginaire émancipateur afin de stimuler les énergies utopiques et de donner un sens aux luttes d'aujourd'hui. Référons-nous ici à l'ouvrage de Dardot et Laval (2014) intitulé Commun qui relie les différentes luttes citoyennes aujourd'hui sur cette terre à une même perspective : l'exigence pour les humains de pouvoir bénéficier des composantes de la vie humaine sans devoir s'esclavagiser, les louer ou les acheter à des possédants en quête de profits permanents et destructeurs de ces mêmes composantes humaines.

Ces composantes, dont nous devons avoir l'usage, sont l'eau, la nourriture, une atmosphère respirable et une terre habitable pour nous et nos enfants, un toit, un revenu, des soins de santé, une vieillesse protégée, les savoirs, les communications et les ressources numériques, l'expression, l'art et les cultures, les espaces verts, etc. Les auteurs désignent par Commun toutes ces aspirations à des usages de vie non conditionnés par des pouvoirs possédants, car le terme «Bien commun » supposerait un possédant.

Dans la même perspective, tout un courant de pensée actuel veut réancrer les villes et les villages, devenus trop souvent des non-lieux : revenir à une nouvelle anthropologie de l'espace, faire de la ville et des lieux de vie en général une communauté de la vie. Privilégier les processus effectifs qui transforment la vie sociale, économique, culturelle et politique. Partir de la vie quotidienne pour se poser des questions concrètes. Par exemple, pourquoi payer l'eau le même prix pour faire son ménage ou remplir sa piscine? Et pourquoi des piscines privées?

41 Il peut se créer des alliances entre plusieurs communes, plusieurs grandes villes. Et pourquoi pas des mouvements plus larges de désobéissance. Durand Folco propose de reconstruire un nouveau système démocratique qui prendrait forme dans les grandes villes et les petits villages également, suggérant ainsi que les communes fassent front commun et créent un rapport de force pour avoir un poids vis-à-vis des décisions prises à différents échelons de pouvoirs, locaux, nationaux ou planétaires (Durand Folco, 2017).

Ancrée dans des pratiques territorialisées, l'approche des intelligences citoyennes a été travaillée localement avec différents collectifs, en Wallonie, à Bruxelles, au Québec, en France, en Amérique latine, en Afrique, débouchant sur des actions qui ont souvent eu un impact sur des responsables politiques et sociaux et sur l'opinion publique. Ainsi des personnes sans logis revendiquent, auprès de la population et des autorités communales, de pouvoir occuper des logements vides et demandent un statut légal pour leur implication dans le quartier, statut qu'elles obtiennent. C'est l'expérience menée à Liège, en Wallonie, à partir d'un atelier d'écriture et d'intelligences citoyennes, atelier abordant aussi le racisme (Eyckmans, Hansotte, 2020).

En Wallonie toujours, à Mouscron, un collectif d'alphabétisation, lié à l'association Lire et Ecrire, envoie aux autorités une lettre dans une langue qui n'existe pas, sollicitant réponse comme si de rien n'était, leur faisant vivre de l'intérieur une situation d'illettrisme, afin que les services communaux prennent en compte cet illettrisme. Une même démarche par le truchement d'une bande dessinée est menée à Nivelles, pour sensibiliser les services postaux et les agents aux guichets à l'humiliation subie par des 
personnes illettrées face à des formulaires compliqués à remplir, et donnés sans ménagements. Dans les deux cas, l'exigence est reconnue et des mesures sont adoptées.

Non directement inscrit dans un processus labellisé "Intelligences citoyennes", mais proche de la manière dont la méthode aborde l'intelligence argumentative, le plaidoyer de femmes boliviennes, péruviennes et sénégalaises, animé par le Monde selon les femmes, revendique un modèle agricole juste et résistant aux destructions multiples (Le Monde selon les femmes, 2015).

\section{Partager un gouvernail pour penser et agir}

Une attitude de base essentielle pour coconstruire une intelligence collective: se donner un outil partagé apte à réguler, à arbitrer, à évaluer. Les humains engagés dans les exigences d'un Commun peuvent se permettre de parler en "Nous", parce qu'ils mettent sur un même pied d'égalité tous les sujets parlants, et qu'ils considèrent que tous les sujets parlants, c'est-à-dire tous les humains, sont pris dans une même destinée, non définie par les pouvoirs verticaux.

Je suis une femme vivant en Somalie. Mon enfant est gravement malade. Quelle est mon espérance ? Je suis père de famille vivant dans une ville du Nord de l'Europe et je ne dispose pas d'un revenu suffisant pour nourrir, soigner, loger, éduquer mes enfants. Quelle est mon exigence ? Je suis une jeune femme en Arabie saoudite. Je souhaite étudier, mener une vie indépendante, je ne veux pas être enfermée. De quoi est-ce que je rêve ? Nous sommes des Syriens migrants, qu'attendons-nous des pays européens? Nous sommes des Palestiniens vivant en territoires occupés. A quoi aspirons-nous? Nous sommes un groupe de femmes (d'ici ou d'ailleurs) violentées dans leur couple. Qu'exigeons-nous de la société où nous vivons? Nous sommes des femmes boliviennes, péruviennes, sénégalaises, confrontées à la sécheresse, à la désappropriation de nos terres et au fait d'assumer seules la survie quotidienne. Que voulons-nous?

L'expression citoyenne et l'intelligence collective relèvent d'une démarche dans laquelle nos interactions sont à trianguler par des repères-tiers qui viennent "entre " nos subjectivités, pour nous amener à construire une parole et une action valides et légitimes. En effet, notre légitimité de citoyennes et citoyens ne repose pas sur un processus électoral. Elle repose sur la qualité et la rigueur de nos procédures d'analyse, d'action, d'intervention. Cette légitimité repose sur un périmètre méthodologique à l'intérieur duquel construire un rapport au Juste (Figure 1), qui peut s'appeler Gouvernail, parce qu'il sert de repère dans la construction et l'ajustement en permanence de nos choix individuels et collectifs (Hansotte, 2013, 2019). 


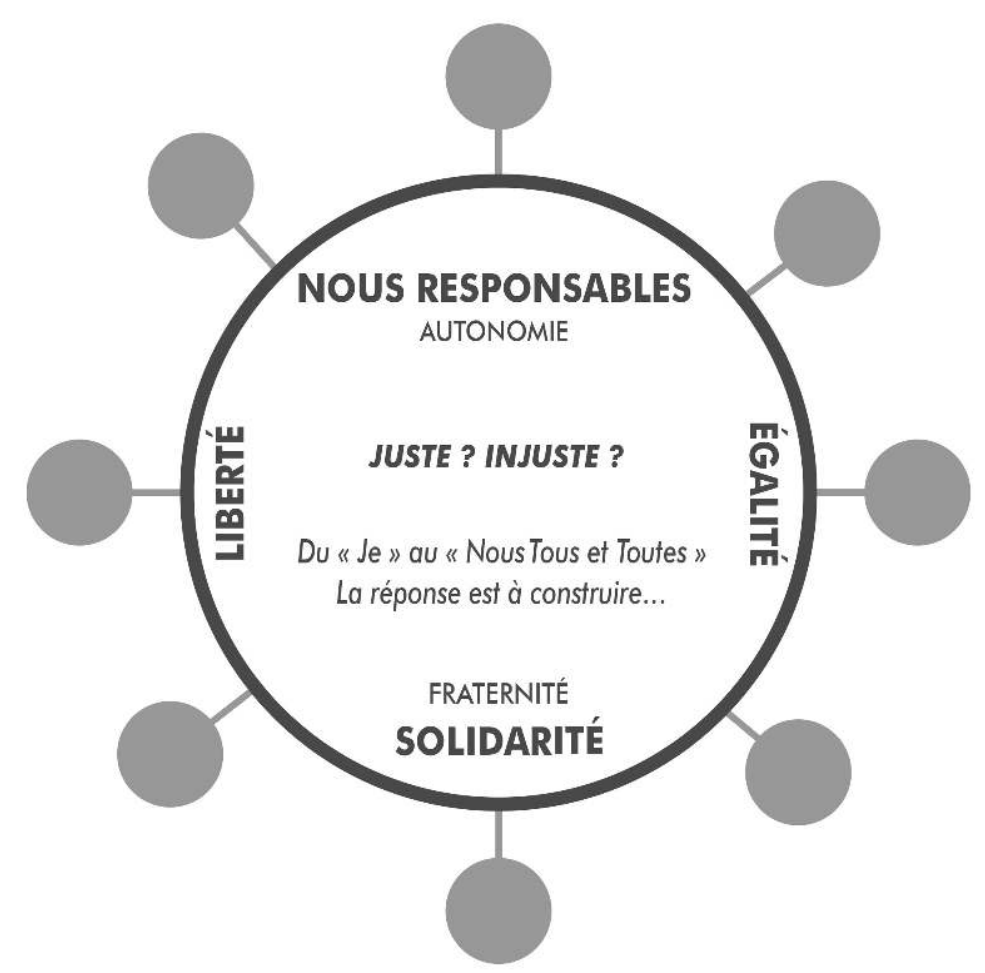

Figure 1. Un gouvernail planétaire

Autonomie (Nous responsables), égalité, liberté, solidarité (impliquant la redistribution des ressources matérielles et immatérielles): ces repères sont considérés dans la tradition des mouvements sociaux comme des visées régulatrices (appelées aussi des principes) qui ne sont pas des vérités révélées par une transcendance. Ces visées ont surgi de l'expérience concrète vécue par des hommes et des femmes au cours de l'Histoire.

C'est la douleur de la non liberté qui rend désirable la liberté, c'est la violence des rapports inégalitaires qui rend forte l'exigence d'égalité, c'est la cruauté d'une société où seuls les riches peuvent se soigner qui rend indispensable l'exigence de solidarité, à travers une redistribution des ressources. Le défi écologique aujourd'hui impose de repenser les inégalités mondiales et locales, les injustices fiscales.

L'accès à une vie digne et juste renvoie à ces 4 visées ou principes fondateurs, pouvant se décliner et s'enrichir à l'infini, lesquels pris tous ensemble constituent un tiers garant. Si l'on parle de visées régulatrices et de gouvernail, c'est qu'il s'agit d'exigences procédurales ou de méthodes par lesquelles s'élaborent progressivement des réponses collectives, à travers tâtonnements, regards et sensibilités multiples. Il ne s'agit pas de réponses a priori.

Chaque fois qu'émerge une situation-problème, la réponse est à construire, en s'appuyant sur les exigences du gouvernail. Une telle attitude distingue la construction des droits politiques, sociaux, humains, la sauvegarde du vivant et la recherche d'une justice écologique de l'affirmation de valeurs issues de codes imposés. Les repères du gouvernail ne représentent pas des réponses a priori face aux situations de la vie. Ils génèrent un questionnement permanent dont les réponses transitoires sont évolutives en fonction des situations. 


\section{Des méthodes pour agir dans l'urgence du vivant}

\section{Transmettre, ressentir, questionner, agir}

51 La difficulté actuelle d'une régulation du chaos, en cours et à venir, invite à s'interroger sur les processus et les méthodes qui permettraient de se réapproprier un pouvoir d'agir.

Les pistes ébauchées dans cet article évoquent la nécessité de se redonner du temps, d'ancrer les démarches d'expérimentation sociale dans des lieux concrets et de travailler dans la perspective d'une articulation des Communs. De même, a été évoquée l'importance d'un gouvernail partagé pour penser et agir

Reste à aborder le principal défi méthodologique d'aujourd'hui : comment faire vivre une conscientisation / éducation à l'urgence du vivant? Comment faire évoluer ce divorce inquiétant, à savoir la déconnexion entre les données informatives, dont nous disposons en permanence, et l'intime volonté de chacun et chacune d'entre nous ? Nous savons mentalement, mais nous semblons ne pas l'avoir intériorisé.

54 L'approche des intelligences citoyennes, inspirée, tout en les renouvelant, des grands courants de l'éducation populaire, peut être vue comme proposant à la fois un modèle heuristique et un parcours expérientiel pour l'agir citoyen. L'approche vise à rendre ce parcours sensible, à travers 4 registres de paroles agissantes, autrement dit 4 registres d'intelligence collective. Ces 4 registres ont traversé l'histoire des luttes, une histoire vis-à-vis de laquelle développer une fidélité inventive. Ces 4 registres sont aussi les conditions futures d'un espace public local et mondial (Hansotte, 2013, 2019). Le Tableau 5 synthétise les principales caractéristiques des intelligences en question. 


\section{Les intelligences citoyennes}

SE RELIER

AFFRONTER

\begin{tabular}{|c|c|}
\hline $\begin{array}{l}\text { Raconter et témoigner } \\
\text { en faveur du Juste }\end{array}$ & $\begin{array}{l}\text { Questionner, } \\
\text { débusquer l'arbitraire, } \\
\text { détourner et inventer }\end{array}$ \\
\hline \multirow[t]{2}{*}{$\begin{array}{l}\text { L'INTELLIGENCE } \\
\text { NARRATIVE }\end{array}$} & $\begin{array}{l}\text { L'INTELLIGENCE } \\
\text { DÉCONSTRUCTIVE }\end{array}$ \\
\hline & ste \\
\hline \multicolumn{2}{|c|}{$\begin{array}{l}\text { Étayer rationnellement ef L'espace public Exiger plus de justice, } \\
\text { collectivement les exigences } \\
\text { revendiquer et proposer }\end{array}$} \\
\hline \multicolumn{2}{|l|}{$\begin{array}{l}\text { Délibérer avec d'autres pour } \\
\text { définir ce qui serait juste }\end{array}$} \\
\hline $\begin{array}{l}\text { L'INTELLIGENCE } \\
\text { ARGUMENTATIVE }\end{array}$ & $\begin{array}{l}\text { L'INTELLIGENCE } \\
\text { PRESCRIPTIVE }\end{array}$ \\
\hline
\end{tabular}

\section{L'intelligence déconstructive}


ultralibéraux et les milieux d'affaires entendent profiter d'un nouveau rapport de force qui leur est favorable pour imposer, avec le soutien des milieux " technoscientistes", une gestion de la crise écologique qui soit profitable aux nantis et fasse payer la facture aux plus pauvres, à travers ce capitalisme vert que vantent les médias (Ariès, 2009, 2017).

62 Ainsi l'idéologie du développement durable permet aux dominants de reprendre la main face à l'urgence environnementale. Comment polluer un peu moins pour polluer plus longtemps? Voire polluer au maximum pour pouvoir dépolluer davantage, à travers une écologie dite "réparatrice "? La solution serait donc uniquement de transformer les consommateurs en consom-acteurs, d'inventer un écocitoyen consumériste.

Autre notion - leurre, le développement durable endort l'esprit critique au nom de la technoscience et de la bonne bouffe. Le développement durable s'inscrit en réalité dans les visées capitalistes, où l'objectif suprême est la maximisation des profits et la croissance infinie (Ariès, 2017).

64 Face à cela, la déconstruction questionne, détourne, subvertit et fait apparaître l'arbitraire. L'urgence dans la démarche des intelligences citoyennes est de cibler « les grands mots » de la "pensée verte ", d'y débusquer les détournements à l'œuvre, de rendre visibles les mensonges et tromperies d'un vocabulaire - simulacre.

\section{L'intelligence narrative}

\section{Ou faire nôtres les défis du devenir}

Le déni ou la démoralisation face aux épreuves du devenir peuvent être interprétés aussi comme une insuffisance de transmission sensible. Les prédictions sur l'évolution de la planète sont ressenties comme un discours théorique extérieur à notre intimité vécue. Pourquoi alors la narration? Il s'agit bien sûr de rompre avec la narration comme pratiques consommatoires (storytellings par exemple, narrations-distractions des réseaux sociaux, voire narrations idéologiques d'endoctrinement).

En quoi les ressources de l'intelligence narrative peuvent-elles être mobilisées au profit d'une réelle écocitoyenneté ? C'est le principe de pacte agissant, responsable et citoyen, qui est à valoriser, invitant le narrateur ou la narratrice à être sincère dans sa narration et ceux ou celles qui écoutent à respecter et à accueillir le récit de l'autre. Une démarche de reconnaissance réciproque. Si quelqu'un raconte quelque chose, c'est parce qu'il est guidé par une certaine espérance ou visée : la narration est actionnée par une recherche de vie bonne ou par la douleur d'un échec (le manque de quelque chose).

Les enjeux écologiques font souvent référence aux violences vécues par d'autres humains, et parfois même par des entités qui apparaissent abstraites, biodiversité ou écosystèmes par exemple, des entités lointaines géographiquement et temporellement. Dès lors, ces violences ne sont pas intériorisées par les citoyens et citoyennes ne les comprenant pas, au sens de prendre avec soi.

69 Ainsi, la difficulté à intégrer dans notre vie les risques planétaires encourus par nous et nos enfants peut être vue comme une faillite de l'intelligence narrative, celle qui fait qu'une situation racontée devient nôtre, est intériorisée, ressentie, perçue et nous amène à bifurquer. L'approche narrative présentée dans les intelligences citoyennes 
encourage des personnes, rassemblées dans un espace de paroles, à raconter une injustice subie, à témoigner de la vie ordinaire.

Il y a toujours une forme d'espérance ou d'exigence en creux dans une démarche narrative : dans un récit, les " héros ", les "gens » subissent et agissent parce qu'ils ont perdu, souffert, et qu'ils veulent vivre autre chose. C'est la personne narratrice qui reconstruit subjectivement son récit et sa quête.

Pour ceux et celles qui accueillent et reçoivent le récit, il s'agit alors de reconnaitre l'injustice ressentie par la personne qui raconte et d'ensuite prendre son histoire avec leur propre parcours de vie, pour mener un travail d'interprétation consistant à faire apparaître le sens dont le récit est porteur, ses ressorts, ses espérances ou visées : un travail herméneutique et non pas un travail de diagnostic objectivant.

Tel est le pacte narratif. Identifier les espérances ou exigences pour le Je qui raconte, et aussi pour le Nous, le groupe qui accueille ce récit et petit à petit, formuler ce qui serait juste pour Nous Tous et Toutes, dégager une visée de changement collectif, sachant que « le Nous Tous et Toutes " n'est jamais physiquement présent. Il s'agit d'une clé éthique et méthodologique, consistant à mettre à l'épreuve du gouvernail nos exigences : ce que nous voulons fait-il avancer, pour nous et les autres humains, les 4 repères du gouvernail (et pas un repère contre les autres) ou les menace-t-il ?

Comment s'élabore ce pacte, cette transmission où la situation vécue par des personnes particulières devient notre situation, nous faisant bifurquer, nous engager et agir? Comment passer de la plainte individuelle à l'exigence collective ? Comment se forment les rapports entre émotion et projet collectif? Comment la peine ou la joie se concilient-elles avec la raison? En fidélité collective au récit, il s'agit de passer du subir à l'agir, à travers tout un processus collectif. Cette démarche, je l'appelle la potentialisation du récit.

74 Cette potentialisation (Tableau 6) s'opère grâce à un travail collectif de résonance au récit, mené en 3 registres, l'affectif, le symbolique, le politique, permettant de mobiliser émotion, invention et exigences de changement. La démarche part des affects qui mettent en mouvement, qui poussent à refuser de subir la situation, affects liés à l'indignation, à la douleur, à la perte de ce à quoi on tient humainement. Le truchement par le symbolique ensuite ouvre la porte à un imaginaire porteur, à des visions autres et valorisables. Et ces deux étapes permettent de formuler des exigences politiques de changements, vers une société juste pour Tous et Toutes, exigences confrontées au gouvernail avant d'être adoptées et suscitant un désir d'engagement. Elles vont générer des actions qui vont se nourrir des trois registres, ce qui leur donnera une force de prescription associant rigueur politique, sensibilité humaine et créativité symbolique. 
Tableau 6 : De la narration à la prescription

\begin{tabular}{|c|c|c|}
\hline $\begin{array}{l}\text { Ce que nous avons } \\
\text { spontanément envie de } \\
\text { dire }\end{array}$ & $\begin{array}{l}\text { Les images qui nous } \\
\text { viennent }\end{array}$ & $\begin{array}{l}\text { Ce que nous voulons } \\
\text { comme changements }\end{array}$ \\
\hline $\begin{array}{l}\text { Une émotion } \\
\text { mobilisatrice }\end{array}$ & Une imagination critique & Un désir transformateur \\
\hline $\begin{array}{l}\text { Cris du cour, questions } \\
\text { brûlantes: }\end{array}$ & Images intérieures : & $\begin{array}{l}\text { Exigences de } \\
\text { changement : }\end{array}$ \\
\hline L'affectif & Le symbolique & Le politique \\
\hline \multirow[t]{4}{*}{$\begin{array}{l}\text { Le récit fait surgir des } \\
\text { paroles de colère, de } \\
\text { tristesse, d'indignation, } \\
\text { des questions } \\
\text { spontanées... }\end{array}$} & $\begin{array}{l}\text { Le récit fait surgir des } \\
\text { visions et images: que } \\
\text { voyons-nous? (Symboles, } \\
\text { objets, personnages, } \\
\text { scènes...) }\end{array}$ & $\begin{array}{l}\text { Le récit interprété par } \\
\text { nous comporte des visées } \\
\text { implicites de changement }\end{array}$ \\
\hline & & $\begin{array}{l}\text { Quels changements } \\
\text { espérés par la personne } \\
\text { narratrice? L'individuel }\end{array}$ \\
\hline & & $\begin{array}{l}\text { Quelles exigences de } \\
\text { changement partageables } \\
\text { par nous qui l'écoutons? } \\
\text { Le collectif }\end{array}$ \\
\hline & & $\begin{array}{l}\text { Confrontation au } \\
\text { gouvernail. Quelles } \\
\text { exigences de changement } \\
\text { à revendiquer pour Nous } \\
\text { Tous et Toutes? La } \\
\text { société et le monde. }\end{array}$ \\
\hline
\end{tabular}

75 Et l'on pourrait valoriser davantage cette intelligence narrative en relayant des récits de souffrances liées aux désastres écologiques, ailleurs et ici, ou en partant de récits d'anticipation, faisant toucher du doigt les dangers à venir, pour les travailler au sein d'un pacte narratif. Le recours au récit et au pacte agissant, triangulé par le gouvernail, est central. Il peut s'agir d'un témoignage vécu, transmis en direct et en synchronie. Il peut s'agir aussi d'un récit fictif ou historique ou encore d'un récit recueilli ailleurs sur la planète, reçu en différé par un groupe qui l'accueille et s'engage dans un agir, en fidélité aux torts subis, aux espérances et exigences des personnes narratrices. Ces transmissions narratives sont à l'œuvre dans de nombreuses mobilisations.

\section{L'intelligence prescriptive}

Ou revendiquer le Juste pour Tous et Toutes

Rendre agissant un récit: une étape active, concrète, revendicative qui favorise un changement collectif. Il s'agit d'entrer dans un registre essentiellement politique, que je nomme intelligence prescriptive. De la narration à l'agir citoyen, la démarche peut déboucher sur des performances valorisant des modalités esthétiques et créatives d'intervention dans l'espace public, pour mobiliser fortement. Parmi ces registres créatifs, que je valorise, reviennent de manière privilégiée l'infiltration, l'installation, le happening, l'image. Le tableau 7 présente deux exemples, extrêmement simples, d'infiltrations activant des prescriptions citoyennes. 


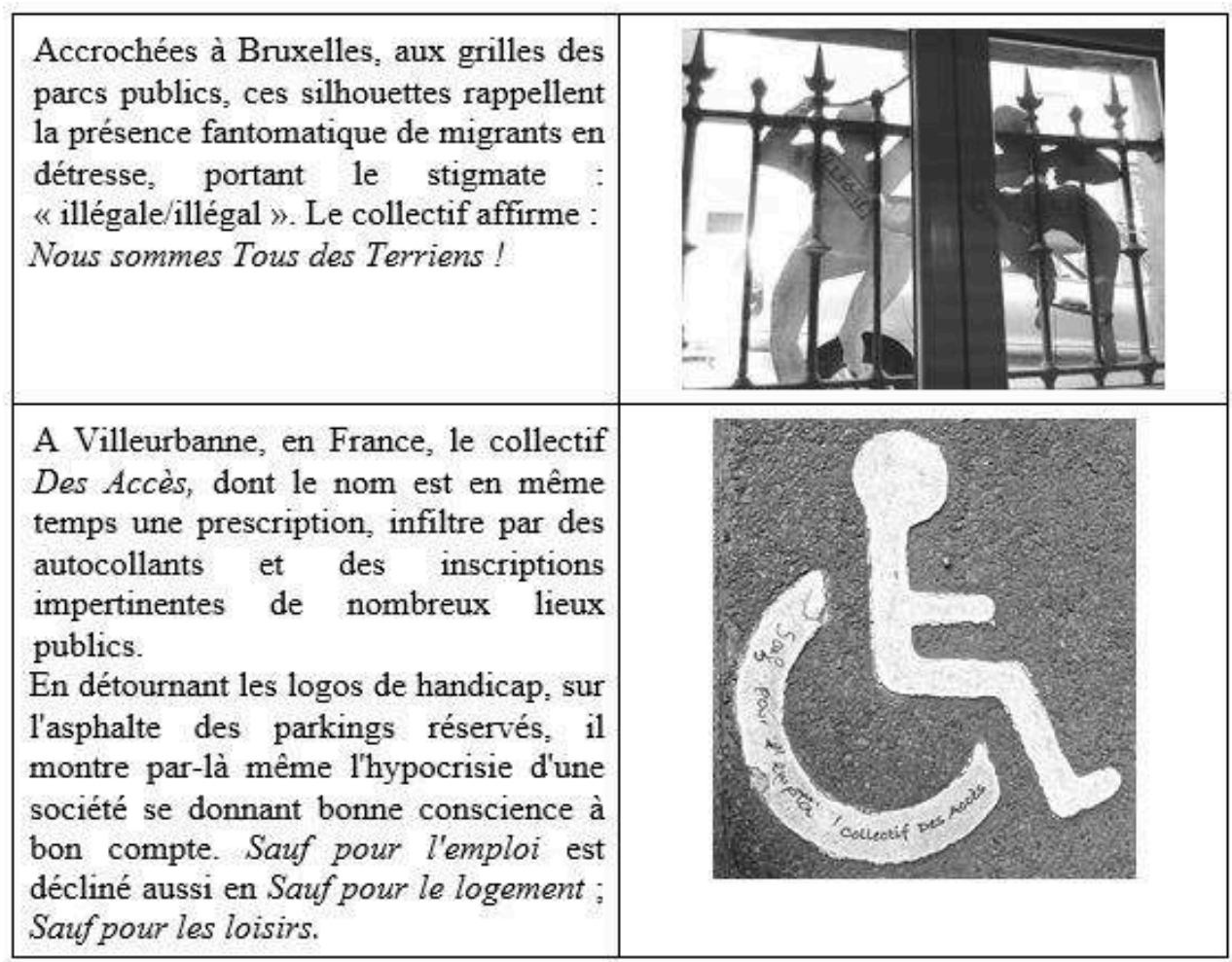

L'étape de la prescription est indispensable à l'émergence d'un mouvement social complet. Être politique, c'est identifier par où passe le devenir dans la situation que nous vivons collectivement. C'est donc l'intelligence du devenir au nom du Commun que les engagements citoyens ont à faire vivre, une intelligence radicale et conflictuelle. L'épreuve de l'énonciation politique devient ici la mise en public d'une exigence, dans une assemblée, une rue ou tout autre lieu, dans les espaces médiatiques. Cette exigence est celle d'une mise en devenir de la situation présente.

\section{L'intelligence argumentative}

Ou étayer des résolutions communes

80 Survient, pour un collectif citoyen, la volonté d'argumenter ce qu'il exige, ce qu'il revendique, ce qu'il propose. L'intelligence argumentative est un moment rationnel dans le déploiement des compétences citoyennes, mais qui doit résulter d'un processus sensible, déconstructif, narratif, prescriptif, et non pas le précéder. Commencer par l'argumentation, c'est empêcher l'imaginaire politique et donner le pouvoir à ceux qui maîtrisent le langage théorique, favorisant une argumentation désincarnée.

Derrière le terme "argumentation" se déploient en réalité deux pratiques très distinctes l'une de l'autre, dont les enjeux diffèrent. Deux orientations différentes et complémentaires structurent le rapport du citoyen à l'argumentation: d'une part argumenter pour soutenir une exigence collective et l'étayer; d'autre part, délibérer avec d'autres pour adopter des résolutions communes.

Abordons en priorité l'argumentation visant à soutenir une exigence collective pour renforcer des positions critiques, des revendications ou propositions, afin de leur donner du poids. Comment le citoyen peut-il donner du poids, de la légitimité et de la 
validité à ses prescriptions sociales, écologiques, à ses exigences d'égalité et d'usage des composantes de la vie humaine, exigences en conflit avec le pouvoir de l'argent?

Une telle compétence est à l'œuvre dans ce qu'on appelle communément le " plaidoyer citoyen » qui se dote d'une rationalité propre. La raison citoyenne en effet ne se confond pas avec la Science tout en s'appuyant sur ses ressources, elle ne se confond pas non plus avec la rationalité comptable, ni avec la rationalité instrumentale, ni avec la rationalité du profit, etc.

\section{Construire une rationalité citoyenne}

Dans la méthodologie des intelligences citoyennes, comment se structure la rationalité d'un plaidoyer? Tout d'abord, ce qui mobilise les citoyens et citoyennes, ce sont les situations vécues par des humains ou par le monde du vivant. Nous nous mettons en mouvement parce que les réalités vécues ne vont pas bien et nécessitent une transformation, une réaction. C'est donc par le vécu qu'un plaidoyer commence, ce vécu qui a été raconté et partagé dans le pacte narratif.

Ensuite, un plaidoyer citoyen se doit de mettre en contexte les situations qui révoltent ou font souffrir, dégageant les déterminants qui pèsent sur les vécus. Le vécu et le contexte nous sont donnés. Il reste à voir, et c'est central dans l'exercice citoyen, ce qui serait plus juste, en valorisant des exigences de changements, des prescriptions politiques. Nous disposons d'un outil de référence commune pour formuler des exigences qui seraient justes pour Tous et Toutes : le gouvernail.

Enfin, un plaidoyer citoyen questionne ce que les lois et les règles en vigueur entretiennent comme injustices. À la lumière de exigences formulées, un plaidoyer citoyen dénoncera les abus de telles lois ou bien proposera de nouvelles règles ou lois.

Le plaidoyer citoyen soutenant des exigences collectives liées au Commun traverse donc 4 registres qu'il articule: un registre expressif et narratif (le vécu), un registre analytique (le contexte), un registre éthico-politique (le juste), un registre juridique (le droit). Le vécu et le juste nous appartiennent pleinement à nous, les citoyens et citoyennes, et non pas aux experts par exemple, et nous devons refuser que l'on nous en dépossède. Le contexte et le droit peuvent nous amener à rechercher des données auprès des ressources humaines ou textuelles d'informations et de savoirs.

L'articulation des 4 registres (Tableaux 8 et 9) donne de la validité à notre plaidoyer. La formulation d'exigences tournées vers le Nous Tous et Toutes lui confère sa légitimité, exigences faillibles et révisibles puisqu'il n'est pas de savoir absolu. 
Tableau 8 : Un plaidoyer citoyen se déploie en 4 registres.

Le vécu - la collecte des narrations vécues ou transmises

"Voilà les situation concrètes, injustes ou intolérables, qui ont motivé notre action ou qui nous mobilisent $»$.

Le contexte -1 'analyse des déterminants qui pèsent sur les situations

"Ces situations s'inscrivent dans tel contexte local ou plus large, contexte social, économique, culturel, géographique. Les faits qui entourent ces situations, les statistiques ou les données informatives éclairent les choses de telle et telle façon ».

Le Juste - la confrontation au gouvernail

Les propositions éthiques et politiques : ce qui serait juste pour Nous Toutes et Tous.

"Voilà ce qui nous paraitrait plus juste en lien avec les situations vécues, le contexte.

Et en fidélité aux droits sociaux, politiques, écologiques, aux principes citoyens 》.

Le Droit - la confrontation aux lois et aux règles

"Les exigences de justice que nous défendons peuvent s'appuyer sur telle disposition légale, nationale ou internationale. Ou bien a contrario, par rapport à nos exigences, le droit national ou international est en défaut et devrait évoluer ».

Tableau 9 : Plaidoyer femmes, violences climatiques (Monde selon les femmes, 2015)

Le vécu:

- La pénurie d'eau, de bois et d'aliments nous oblige à aller loin pour chercher l'indispensable.

- Les hommes ne partagent pas les tâches domestiques avec nous et c'est aggravé par le changement climatique.

- Les fermes qui vivent dans les villes sont exploitées et subissent des sévices sexuels.

- Les pratiques de répartition nous attribuent les parcelles les moins fertiles.

Le contexte:

- Inondations, sécheresse, gréle et diminution de la production.

- Perte des semences ancestrales et dépendance aux multinationales.

- Maladies, parasites, manque d'eau et mort d'animaux.

- Système capitaliste et croissance sans limite.

Le juste:

- Défense d'une pêche artisanale tournée vers la consommation locale.

- Redistribution des tâches productives entre époux pour accroître nos pouvoirs de décision.

- Élaboration de systèmes d'irrigation communautaires.

- Recherches-actions pour adapter nos savoirs traditionnels en matière de semences pour sauver la biodiversité.

- Accès pour les femmes aux bonnes terres, à leur contrôle et à la propriété foncière.

Le droit :

- Droit de s'organiser en syndicats et groupements de femmes.

- Coresponsabilité hommes-femmes dans tous les domaines.

- Équité de droit dans l'accès aux ressources.

\section{Pratiquer une délibération démocratique}

89 Parmi les pratiques propres à l'espace public, on trouve l'exigence d'une délibération argumentée entre citoyens et citoyennes. Et pourtant le débat, outil de citoyenneté, figure parmi les pratiques citoyennes les plus corrompues : un jeu de gladiateurs. On est séduit ou attiré par la joute plutôt que par la recherche et l'interrogation. Au détriment de l'intelligence collective, les actes de paroles s'entrechoquent selon une 
compétition interpersonnelle: persifler, intimider, convaincre, séduire, amuser, déstabiliser, ridiculiser, dénier, disqualifier, soupçonner, etc. repères proposés dans cet article. Dans le pacte narratif, comment accueillir les témoignages non humains, faire surgir leur récit et les prendre avec soi, dans la démarche de compréhension et de potentialisation? Dans la délibération, comment faire intervenir des voix représentant ces non humains, ne plus les considérer comme un simple contexte de plaidoyer? Tel est le défi méthodologique, éthique et politique qui s'ouvre dans la mise en œuvre aujourd'hui des intelligences citoyennes.

\section{BIBLIOGRAPHIE}

Ariès, P. (2017). Désobéir et grandir. Montréal : Les Éditions Écosociété.

Eyckmans, G. et Hansotte, M. (2020). Un Destin. Nos destinées. Liège : Édition Delfi.

Dardot, P. et Laval, C. (2014). Commun : essai sur la révolution au XXIe siècle. Paris : La Découverte.

Durand Folco, J. (2017). À nous la ville! Traité de municipalisme. Montréal : Les Éditions Écosociété. 
Hansotte, M. (2008). Les intelligences citoyennes. Bruxelles : De Boeck Université.

Hansotte, M. (2013). Mettre en œuvre les intelligences citoyennes. Bruxelles : Édition Le monde selon les femmes.

Hansotte, M. (2019). Juste? Injuste? Activer les intelligences citoyennes. Liège : Édition Delfi.

Latour (B.), 2004, Politiques de la nature. Comment faire entrer les sciences en démocratie. Paris : Édition La Découverte, collection « Sciences humaines et sociales ».

Morlaye, D. et Charlier, S. (2015). Stratégies de femmes face au changement climatique in Recherche et plaidoyer. Bruxelles : Édition Le Monde selon les femmes.

Stiegler (B.), 2016, Dans la disruption. Comment ne pas devenir fou ? Paris : Les liens qui libèrent.

\section{RÉSUMÉS}

Désastres écologiques, migrations, violences, inégalités. Tout se passe comme si les acteurs et actrices démocratiques n'arrivaient plus à réguler les évolutions de ce monde. Comment réinventer alors une nouvelle forme de puissance publique? Comment reconstruire une intelligence collective, un pouvoir avec, pour susciter une prise de conscience, une mobilisation? Nous sommes dans une bifurcation historique, car s'annonce une aventure indéterminée où la régulation citoyenne aurait en responsabilité l'urgence du vivant. La méthodologie dégagée dans la recherche sur les Intelligences citoyennes tente de répondre à ce défi en favorisant le passage du subir à l'agir, à travers un modèle heuristique d'action collective faisant vivre un espace public, local et mondial. L'intelligence narrative est celle qui nous rend capables de raconter des situations injustes et destructrices, d'en témoigner pour amener une réaction, un engagement agissant. Au-delà, s'impose l'intelligence déconstructive: résister aux leurres de la pensée dominante, les détricoter. Un collectif citoyen engagé dans les questions de justice sociale et de sauvegarde du vivant doit pouvoir ensuite construire collectivement des propositions alternatives visant la réappropriation humaine des communs : c'est l'intelligence prescriptive. Cette construction collective passe enfin par l'intelligence argumentative, qui nous rend capables d'argumenter nos exigences et de délibérer de façon démocratique. Le déploiement de ces quatre intelligences nécessite des démarches et des processus d'éducation populaire.

Ecological disasters, migrations, violence, inequalities. All happen as if democratic actors no longer succeed in regulating the evolutions in which they are caught. How to reinvent a new form of societal empowerment? How to rebuild a collective intelligence in order to create an awakening, a call to action? We are at historical crossroads with an unknown future for societal self-regulation and its responsibility for survival of life in all its forms. The methodology developed in the research « Citizen Intelligences » attempt to handle this challenge by promoting action instead of passivity through a heuristic model of collective action for the survival of local and global public space. Narrative intelligence allows the telling of unjust and destructive situations to create a reaction and an active involvement. It must be combined with the deconstructive intelligence which means dealing with the threats of the dominant thought process by removing one layer at the time. A group of people concerned with social justice and protection of life must then work together to build options in order to reallocate to all people the common components of life. That is called the prescriptive intelligence. This collective construction finally goes through the argumentative intelligence which enables us to present the arguments for our requirements and to deliberate democratically. The deployment of these four intelligences requires processes of popular education. 
INDEX

Mots-clés : citoyenneté, intelligence citoyenne, justice sociale, démocratie, intelligence

Keywords : citizenship, citizen intelligence, social justice, democracy, intelligence

\section{AUTEUR}

\section{MAJO HANSOTTE}

Majo Hansotte est l'auteure d'une thèse sur l'espace public contemporain intitulée « Les intelligences citoyennes » (Hansotte 2008). Elle a réalisé plusieurs documents méthodologiques en lien avec des préoccupations de terrain. Sa démarche de formation s'adresse à des acteurs et actrices de l'éducation populaire, à des citoyens et citoyennes. L'enjeu de la formation tourne autour de la capacité à dire le Juste et l'Injuste pour agir à travers des intelligences collectives. 